\section{Robotic double-loop reconstruction method following total gastrectomy}
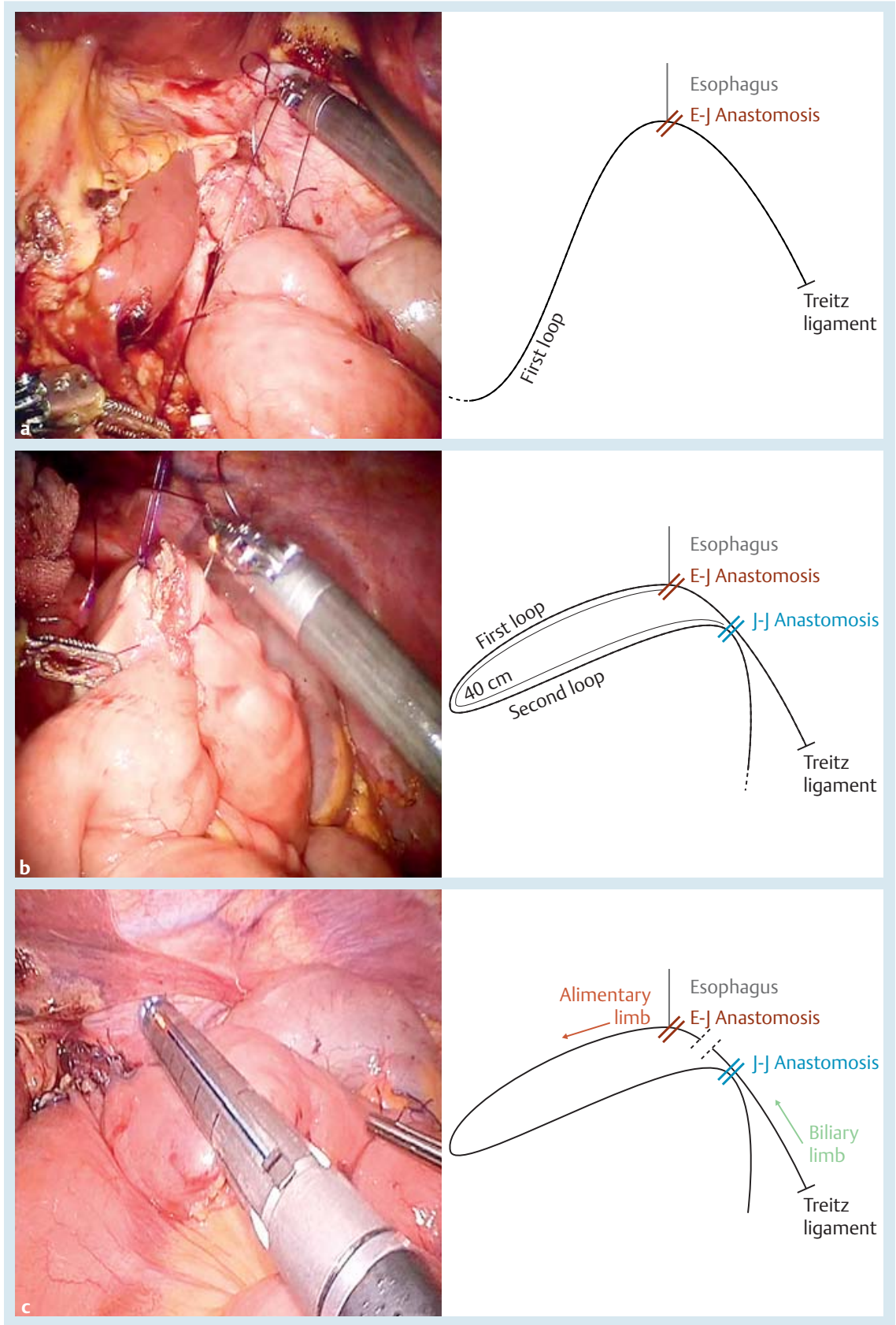

Fig. 1 Photographs and schematics showing: a the first loop, which allows the creation of the esophagojejunal (E-J) anastomosis; $\mathbf{b}$ the jejuno-jejunal (J-J) anastomosis, which is created between the alimentary and biliary limbs on the left side of the first anastomosis; $\boldsymbol{c}$ the interruption between the E-J and $\mathrm{J}-\mathrm{J}$ anastomoses, using a linear stapler, that converts the procedure into a modified Roux-en-Y.

Minimally invasive surgery for gastric cancer is a challenge. The reconstructive time is a particular issue and researchers have adopted a large variety of solutions and produced heterogeneous data.
The reconstructive phase can be divided into two major categories based on the approach adopted: the execution of extracorporeal versus intracorporeal anastomosis. In turn, the surgical team can perform the latter with laparoscopic or ro-

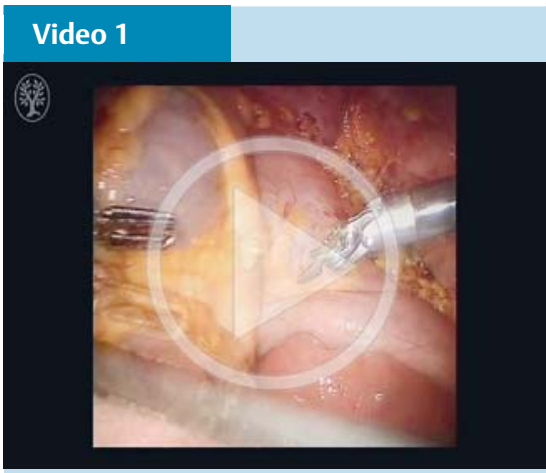

The double-loop reconstruction method with intracorporeal robot-sewn anastomoses: the selected intestinal segment is joined to the esophagus (becoming the first loop); an endto-side esophagojejunal robot-sewn anastomosis is created; the second loop is formed from the alimentary limb, which is placed close to the first anastomosis, defining the jejuno-jejunal anastomosis; finally a mechanical stapler is used to interrupt the continuity of the bowel between the two anastomoses.

botic assistance. However, the question is, how should a robotic esophagojejunal anastomosis be performed after total gastrectomy?

Most articles in the literature have reported the execution of mechanical anastomoses [1-6], especially with circular staplers via the creation of a manual purse-string around the anvil. Other solutions have described the use of the Orvil or the overlap technique. Only three authors have reported intracorporeal sutures with a completely robotic-sewn anastomosis [7-9].

A new robotic technique (the Parisi technique) was developed and adopted at St. Mary's Hospital, Terni, Italy. A doubleloop reconstruction method with an intracorporeal robot-sewn anastomosis is performed $(\bullet$ Video 1$)$.

A selected intestinal segment, coming from the ligament of Treitz, must be free of tension or torsion. The small intestine is then joined to the esophagus with two stitches that pair the esophageal angles to the selected loop so as to locate the biliary side to the left of the esophagus and the alimentary side to the right. This represents the first loop ( $\bullet$ Fig. 1 a).

Now, the surgeon can perform an end-toside esophagojejunal robot-sewn anasto- 
mosis. Initially, the suturing begins on the posterior margin. The external posterior layer is sutured with interrupted stitches taking the jejunal serosa and the esophageal muscle fibers. The small intestine is then opened as well as the end of the esophagus.

The internal posterior layer is sutured by either an interrupted or a continuous suture from one angle to the opposite one. The jejunum and the esophagus are now well matched and the internal suture continues on the anterior plane. Next, suturing of the external anterior layer is performed with interrupted stitches to complete the esophagojejunal anastomosis. The route of the alimentary limb is followed upward to reach a distance of about $30-40 \mathrm{~cm}$ from the esophagojejunal anastomosis. In this way, the bowel that will form the second loop is identified. It is carried upward, avoiding intestinal twisting, and placed close to the first anastomosis ( $\bullet$ Fig. 1 b). The jejuno-jejunal anastomosis is then created using a mechanical stapler fired by an assistant. The opening is closed with a robotic suture.

The last step of the procedure is the interruption of continuity between the esophagojejunal and jejuno-jejunal anastomoses to create the RouX-en-Y, by firing the linear stapler ( $\bullet$ Fig. 1 c).

Endoscopy_UCTN_Code_TTT_1AT_2AC

Competing interests: None

\section{Amilcare Parisi ${ }^{1}$, Stefano Trastulli ${ }^{1}$, Francesco Ricci ${ }^{1}$, Roberto Cirocchi ${ }^{1}$, Daniele Pironi ${ }^{2}$, Alberto Santoro ${ }^{2}$, Jacopo Desiderio ${ }^{1}$}

${ }^{1}$ Department of Digestive Surgery, St. Mary's Hospital, University of Perugia, Terni, Italy

${ }^{2}$ Department of Surgical Sciences, Sapienza University of Rome, Rome, Italy

\section{References}

1 Desiderio J, Jiang ZW, Nguyen NT et al. Robotic, laparoscopic and open surgery for gastric cancer compared on surgical, clinical and oncological outcomes: a multi-institutional chart review. A study protocol of the International study group on Minimally Invasive surgery for GASTRIc Cancer-IMIGASTRIC. BMJ Open 2015; 5: e008198

2 Parisi A, Desiderio J. Establishing a multi-institutional registry to compare the outcomes of robotic, laparoscopic, and open surgery for gastric cancer. Surgery 2015; 157: 830-831

3 Parisi A, Nguyen NT, Reim D et al. Current status of minimally invasive surgery for gastric cancer: A literature review to highlight studies limits. Int J Surg 2015; 17: 34-40

4 Son T, Lee JH, Kim YM et al. Robotic spleenpreserving total gastrectomy for gastric cancer: comparison with conventional laparoscopic procedure. Surg Endosc 2014; 28: 2606-2615

5 Yoon HM, Kim YW, Lee JH et al. Robot-assisted total gastrectomy is comparable with laparoscopically assisted total gastrectomy for early gastric cancer. Surg Endosc 2012; 26: $1377-1381$
6 Hur H, Kim JY, Cho YK et al. Technical feasibility of robot-sewn anastomosis in robotic surgery for gastric cancer. J Laparoendosc Adv Surg Tech A 2010; 20: 693-697

7 Jiang ZW, Liu J, Wang G et al. Esophagojejunostomy reconstruction using a robot-sewing technique during totally robotic total gastrectomy for gastric cancer. Hepatogastroenterology 2015; 62: 323-326

8 Liu XX, Jiang ZW, Chen $P$ et al. Full robotassisted gastrectomy with intracorporeal robot-sewn anastomosis produces satisfying outcomes. World J Gastroenterol 2013; 19: 6427-6437

9 Parisi A, Ricci F, Trastulli S et al. Robotic total gastrectomy with intracorporeal robotsewn anastomosis: a novel approach adopting the double-loop reconstruction method. Medicine (Baltimore) 2015; 94: e1922

\section{Bibliography}

Dol http://dx.doi.org/

10.1055/s-0042-101386

Endoscopy 2016; 48: E55-E56

(c) Georg Thieme Verlag KG

Stuttgart · New York

ISSN 0013-726X

\section{Corresponding author}

Jacopo Desiderio, MD

Department of Digestive Surgery

St. Mary's Hospital

University of Perugia

Tern

Italy

djdesi85@hotmail.it 